BMC

Bioinformatics

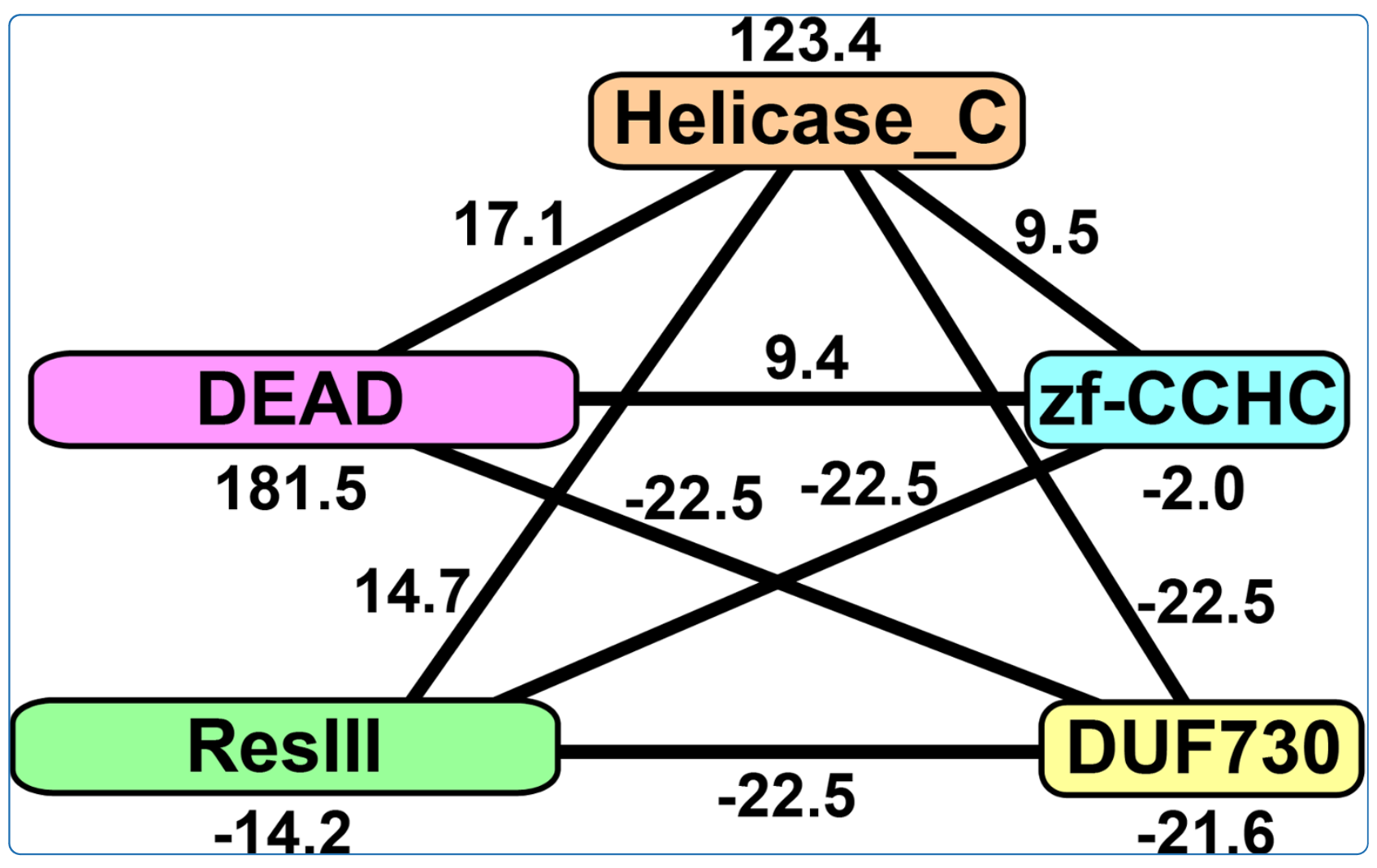

Using context to improve protein domain identification

Ochoa et al.

C Biomed Central 


\title{
Using context to improve protein domain identification
}

Alejandro Ochoa ${ }^{1,3}$, Manuel Llinás ${ }^{1,3^{*}}$ and Mona Singh $2,3^{*}$

\begin{abstract}
Background: Identifying domains in protein sequences is an important step in protein structural and functional annotation. Existing domain recognition methods typically evaluate each domain prediction independently of the rest. However, the majority of proteins are multidomain, and pairwise domain co-occurrences are highly specific and non-transitive.

Results: Here, we demonstrate how to exploit domain co-occurrence to boost weak domain predictions that appear in previously observed combinations, while penalizing higher confidence domains if such combinations have never been observed. Our framework, Domain Prediction Using Context (dPUC), incorporates pairwise "context" scores between domains, along with traditional domain scores and thresholds, and improves domain prediction across a variety of organisms from bacteria to protozoa and metazoa. Among the genomes we tested, $\mathrm{dPUC}$ is most successful at improving predictions for the poorly-annotated malaria parasite Plasmodium falciparum, for which over 38\% of the genome is currently unannotated. Our approach enables high-confidence annotations in this organism and the identification of orthologs to many core machinery proteins conserved in all eukaryotes, including those involved in ribosomal assembly and other RNA processing events, which surprisingly had not been previously known.

Conclusions: Overall, our results demonstrate that this new context-based approach will provide significant improvements in domain and function prediction, especially for poorly understood genomes for which the need for additional annotations is greatest. Source code for the algorithm is available under a GPL open source license at http://compbio.cs.princeton.edu/dpuc/. Pre-computed results for our test organisms and a web server are also available at that location.
\end{abstract}

\section{Background}

Protein domains are fundamental units of protein structure, function, and evolution. As a result, domain prediction is an important first step in the annotation of protein sequences [1]. Enhancements in domain identification improve protein annotations, as domains are often associated with specific cellular functions, and novel domain predictions can either newly predict or further refine functional predictions [2,3]. Furthermore, some domains are known to be associated with structures and thus their identification can be used for inferring protein structure $[4,5]$. Domain predictions are also the starting point for a range of more sophisticated

\footnotetext{
* Correspondence: manuel@genomics.princeton.edu; mona@cs.princeton.edu 'Department of Molecular Biology, Princeton University, Princeton, USA ${ }^{2}$ Department of Computer Science, Princeton University, Princeton, USA Full list of author information is available at the end of the article
}

analyses, including comparative genomics of domain families in diverse organisms [6-8], studies of the evolution of protein and domain structure and function [9-11], prediction of protein-protein interactions [12-15] and identification of complex evolutionary relationships [16].

The majority of proteins contain more than one domain [17]. Domains occur in different combinations, and the domain composition of multidomain proteins is critical for their specialized functions. Domains do not form random combinations, and indeed a limited fraction of domain pairs and triplets are highly recurrent [18]. While the mechanisms that lead to new domain combinations have been extensively explored and the analysis of observed domain combinations has received significant recent attention (reviewed in [19]), this
C Biomed Central

() 2011 Ochoa et al; licensee BioMed Central Ltd. This is an Open Access article distributed under the terms of the Creative Commons Attribution License (http://creativecommons.org/licenses/by/2.0), which permits unrestricted use, distribution, and reproduction in any medium, provided the original work is properly cited. 
information has not yet been widely used for domain prediction.

Here, we exploit the tendency of certain domains to co-occur with each other in order to improve domain identification. While domains within any given sequence are typically identified by considering each domain family individually, domain co-occurrence or "context" is useful in detecting weak sequence similarity [20]. In particular, two domain families that frequently co-occur provide "positive context", and for a given sequence, if these domains are identified with low confidence individually, their weak signal can be amplified. Similarly, domain family pairs that have never been observed provide "negative context" and their occurrences can be penalized (but not necessarily eliminated), thereby preventing unnatural combinations of low scoring predictions and limiting false predictions.

We have developed a novel graph-theoretic framework that combines individual domain scores with pairwise scores derived from domain co-occurrence statistics, in order to find a set of domains that maximize an overall score. Our approach, dPUC (Domain Prediction Using Context), uses Pfam [21] profile hidden Markov models (HMMs) [22] to score domains individually, along with a novel log-odds scoring system that captures the propensity of pairs of domains to be found in the same sequence. While we have developed our approach using Pfam, alternate libraries of domain profiles (e.g., SMART [23], Superfamily [4], or CDD [5]), as well as different pairwise context scoring schemes, can be readily incorporated.

We test dPUC via rigorous benchmarks on eight organisms, ranging from bacteria (Escherichia coli and Mycobacterium tuberculosis), to protozoa (yeast and Plasmodium species) and metazoa (human, fly, worm). We present the first large-scale demonstration that incorporating domain context improves domain predictions in organisms across the evolutionary spectrum. Overall, dPUC gains up to $11 \%$ more domains at noise rates comparable to the Standard Pfam's, and outperforms the recently-published method CODD (CoOccurent Domain Discovery) which also incorporates domain context [24]. Further analysis demonstrates that dPUC's performance improvements are due in part to penalization of negative context as well as allowing context between repeated domains. Importantly, we also find that dPUC does not require much additional time beyond that necessary for Pfam to initially identify domains.

We have found that dPUC is particularly effective at improving domain predictions for the genome of the poorly annotated malaria parasite Plasmodium falciparum. For this parasite, we perform further testing and show that dPUC's predictions are consistent among orthologs in closely related Plasmodium species. Moreover, we have used dPUC to annotate Plasmodium proteins and have newly identified proteins taking part in core processes such as ribosomal assembly and other RNA processing events. Overall, our findings in Plasmodium and other genomes suggest that domain identification can be significantly improved by incorporating context, particularly for organisms with poorly understood genomes.

\section{Methods \\ Data}

\section{Pfam database}

Pfam 23 was downloaded from the website (http://pfam. sanger.ac.uk/). A list of nesting families was extracted from Pfam-A.seed, defining a set of "allowed overlaps" consisting of domains with overlapping amino acid ranges within a protein sequence. The domain architecture of a protein is defined as its ordered list of domains, including repeats. We parsed the Pfam-A.full file to obtain the complete domain assignments to Uniprot 12.5 sequences, and thereby the domain architectures used to compute domain context scores (described below).

\section{Proteomes}

We used the proteomes of several model organisms for our testing, along with those of several parasites of medical interest. The proteomes of E. coli, M. tuberculosis, Saccharomyces cerevisiae, Caenorhabditis elegans, Drosophila melanogaster, and Homo sapiens, were downloaded from Uniprot [25] 15.8 (15.10 for $M$. tuberculosis), by obtaining all sequences with the organism taxon numbers 83333, 1773, 4932, 6239, 7227, and 9606, respectively, the keyword "complete proteome" (keyword 181), and not including isoforms. The proteomes of the Plasmodium species P. falciparum [26], and $P$. vivax [27] were downloaded from PlasmoDB 6.0 [28], and sequences containing internal stop codons were removed. Consistency of predictions on the Plasmodium species were further tested by analysis on $P$. knowlesi [29], P. chabaudi, P. berghei, and P. yoelii, which were also downloaded from PlasmoDB.

\section{Approach}

\section{The dPUC model}

For a given protein sequence, let $P$ be a set of candidate domains (which we obtain by setting a permissive threshold on the HMMER domain E-values). For each domain $i$ in $P$, let $H_{i}$ be the domain score of $i$, let $T_{i}$ be the domain score threshold for the family of domain $i$, and for each pair of domains $i$ and $j$, let $C_{i j}$ be the context score between $i$ and $j$ (see dPUC context scores below). Let $D \subseteq P$ be a subset of domains, and then for 
each domain $i$ we define its score with respect to this set of domains as

$$
S_{i, D}=H_{i}-T_{i}+\Sigma_{j \in D} C_{i j} .
$$

Our goal is to find the subset of domains $D \subseteq P$ that maximizes the "total score" $\Sigma_{i \in D} S_{i, D}$, constrained so that $D$ only contains "allowed overlaps" and each domain $i$ in D satisfies the domain threshold $S_{i, D} \geq 0$. Note that without context scores (that is, $C_{i j}=O \forall i, j$ ), the last inequality is reduced to the standard definition of a domain threshold. Our framework can be illustrated from a graph-theoretic point of view (Figure 1).

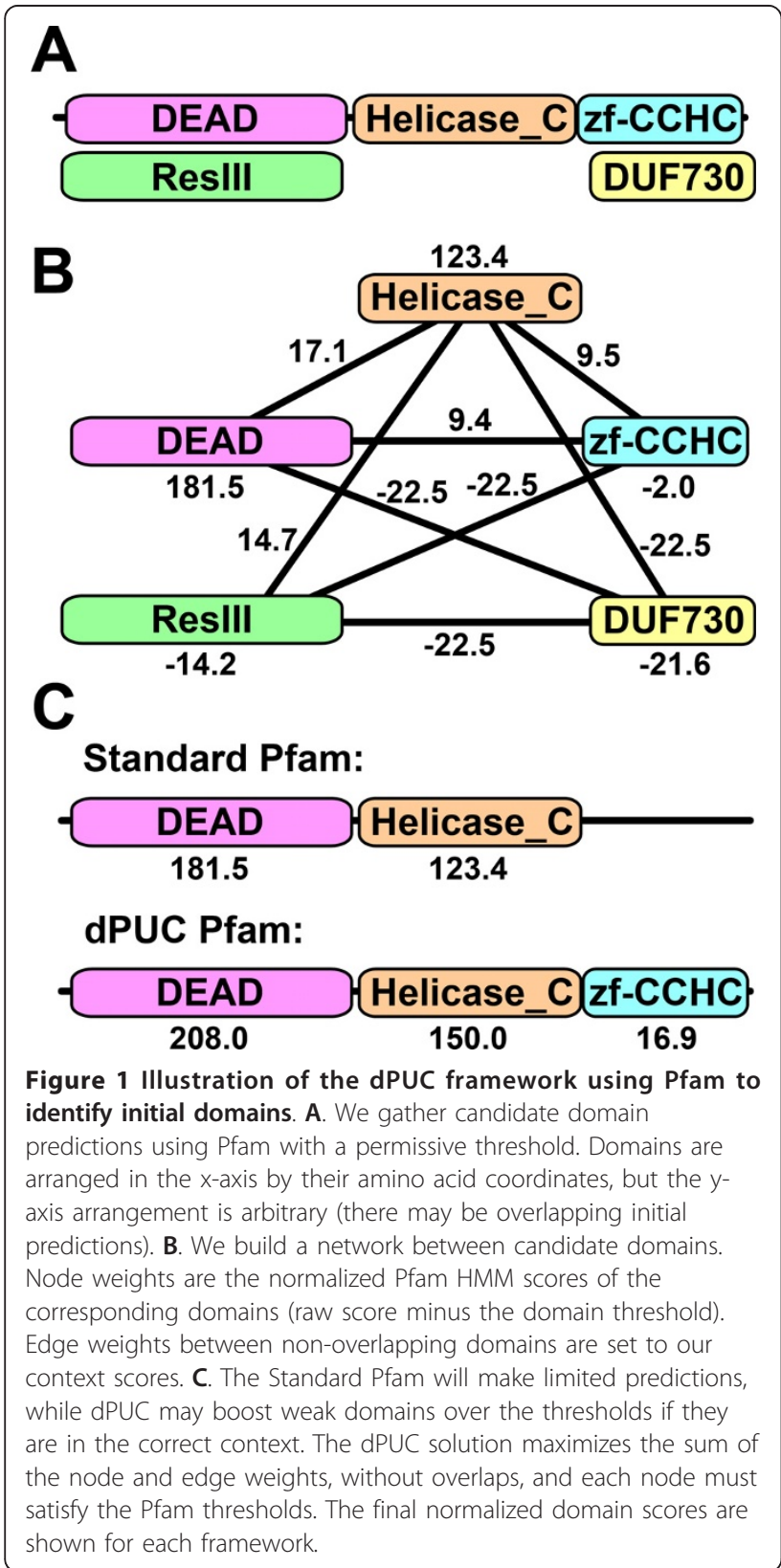

\section{Formulation using ILP}

We solve our combinatorial optimization problem with integer linear programming (ILP). In particular, we define a $0 / 1$ variable $x_{i}$ for each domain $i$, and a $0 / 1$ variable $x_{i j}$ for each pair domains $i$ and $j$. If variable $x_{i}$ is set to 1 , this indicates that domain $i$ is included in our final set of predictions. Similarly, if variable $x_{i j}$ is set to 1 , this indicates that the pair of domains $i$ and $j$ are in our final set of predictions. The score of each domain with respect to the other chosen domains is given by

$$
S_{i}=\left[H_{i}-T_{i}\right] x_{i}+\Sigma_{j} C_{i j} x_{i j} .
$$

Our goal is to maximize

$$
\begin{array}{ll}
\Sigma_{i} S_{i} \text { such that } & \\
x_{i}, x_{j}, x_{i j} \in\{0,1\} & \forall i, j, \\
0 \leq x_{i}+x_{j}-2 x_{i j} \leq 1 & \forall i, j\left(\text { enforces } x_{i j}=x_{i} \& x_{i j}=x_{j}\right), \\
x_{i}+x_{j} \leq 1 & \forall i, j \text { with disallowed overlaps, } \\
S_{i} \geq 0 & \forall i \text { (enforces domain threshold), and } \\
\Sigma_{i \in F} S_{i}+T_{i} x_{i} \geq T_{F}{ }^{*} & \forall \text { families } F \text { (enforces a Pfam threshold), }
\end{array}
$$

where $T_{F}^{*}$ is a Pfam-specific sequence threshold (see Additional File 1 "dPUC implementation details"). Though a theoretically difficult problem, we solve this ILP via a two pronged approach based on domain elimination and using lp_solve 5.5.0.14 [30]. See Additional File 1 Supplementary Methods for further information on solving the ILP and relevant details about the Pfam curated and domain-specific "gathering" thresholds, including the treatment of combined local and glocal domains.

\section{dPUC context scores}

We derive pairwise log-odds context scores using Pfam architectures found in Uniprot. These architectures are filtered to remove those that occurred in only one sequence, since they are more likely to be erroneous. Let $e_{i p}$ be the number of domains of family $i$ in protein $p$, and let $e_{p}$ be the total number of domains in protein $p$. We obtain "normalized pair counts" $c_{i j}$ as follows:

$$
\begin{array}{ll}
c_{i j}=\Sigma_{p} e_{i p} e_{j p} /\left(e_{p}-1\right) & \forall i, j \text { if } i \neq j, \text { and } \\
c_{i i}=\Sigma_{p} e_{i p}\left(e_{i p}-1\right) /\left(e_{p}-1\right) & \forall i,
\end{array}
$$

where the sum over proteins $p$ goes only over multidomain proteins, as single domain proteins would have zero denominators. Note that the normalization given above divides the pairs from each protein by the total number of domains of that protein (minus one), to compensate for inflated counts due to proteins with many domains. While there are many possible normalization schemes, our scheme keeps domains from counting themselves, and each protein $p$ contributes $e_{p}$ counts to all $c_{i j}$ in total. These counts are turned into probabilities by setting

$$
p_{i j}=\left(c_{i j}+\alpha / n^{2}\right) /(c+\alpha) \quad \forall i, j,
$$


where $c=\Sigma_{i j} c_{i j}, \alpha=1$ is the regularization parameter (to handle the zero count case), and $n$ is the number of domain families observed in the architectures $(\leq 10,340$ families in Pfam 23 due to architecture filtering). By construction, $\Sigma_{i j} p_{i j}=1$. The null model probabilities are

$$
p_{i}=1 / n \quad \forall i,
$$

since random domains appear approximately uniformly using $E$-value thresholds. The context scores are

$$
C_{i j}=\log _{b}\left[p_{i j} /\left(p_{i} p_{j}\right)\right] \forall i, j,
$$

with $b=2$, to match the HMMER2 bit scores. From these equations we can derive that unobserved pairs (pairs with $c_{i j}=0$ ) are always penalized, since they are assigned the same large negative score of $-\log _{b}[1+c / \alpha]$. Finally, observed pairs with negative scores are instead set to zero. This way, observed pairs are never penalized. For Pfam 23, only 5 out of the 15,929 observed pairs had negative scores that were subsequently set to zero. We also experimented with other log-odds scoring schemes (see Additional File 1 Supplementary Results); however, most schemes did not significantly change the performance of the overall approach.

\section{Empirical analysis of dPUC runtimes}

Since each protein sequence is a separate problem, the problem can be parallelized over proteins. We considered the 25,047 proteins of E. coli, M. tuberculosis, S. cerevisiae, and $P$. falciparum, and ran each problem on a $2.66 \mathrm{GHz}$ Intel processor with $8 \mathrm{~GB}$ RAM. Runtime is measured in wall clock time. The Standard Pfam (the bulk of which is HMMER processing) runs in 96.4 \pm 41.4 seconds per protein (mean \pm standard deviation), while the dPUC overhead (that is, not including HMMER) runs at an additional $0.0229 \pm 0.865 \mathrm{~s} / \mathrm{pro}-$ tein. However, the runtime distributions have very long tails, as can be seen in Additional File 1 Figure S1. Nevertheless, we find that in $95 \%$ of the cases, the dPUC optimal solution is obtained within $0.015 \mathrm{~s}$, and in $99.94 \%$ of the cases, the optimal solution is found within $1 \mathrm{~s}$.

\section{Other approaches \\ Baseline methods}

We tested three non-context methods that serve as natural baselines for dPUC. The first is the "Standard Pfam", which uses their curated and domain-specific "gathering" (GA) thresholds (details in Additional File 1 "Pfam relevant details"). Since the Standard Pfam produces a single data point, we created the "Pfam Extended GA" method, in which the GA thresholds are shifted by constant amounts (details in Additional File 1 "Pfam Extended GA thresholds"), allowing us to explore a range of noise cutoffs. Lastly, the "Pfam $E$-value" method uses domain $E$-value thresholds instead of the GA thresholds.

\section{CODD context method}

To contrast with dPUC, we implemented two simple context approaches that filter candidate domains. The first is based on CODD, which incorporates positive context information whereby a low scoring domain can be predicted based on co-occurrence with a higher scoring domain [24]. Given a network of positive context domain pairs, a set $G$ of Pfam predictions that pass the gathering thresholds with no disallowed overlaps, and a set of candidate predictions $D$, this filter finds the set of final predictions $P$ as follows. First we initialize $P=G$. For each domain $d$ in $D$ sorted ascending by $E$-value, we transfer $d$ to $P$ if $d$ has positive context with any domain in $G$ and $d$ does not have disallowed overlaps with any domains in $P$. The second approach is a novel double positive and negative filter of our creation that we call nCODD, and is described in the Additional File 1 Supplementary Methods.

\section{Testing}

We developed two approaches for assessing the performance of dPUC.

\section{Estimated FDR}

In our first test, we compare the number of predictions from different methods (i.e., Standard Pfam, dPUC, and CODD) on real and shuffled protein sequences. The key idea is that domain predictions on shuffled sequences arise by chance alone, whereas predictions on real sequences give us the total number of predictions (true or false), and their ratio approximates the false discovery rate. We shuffle the residues of each protein separately, thereby preserving the amino acid composition of each sequence as well as the length distribution over each proteome. For the context methods (dPUC, CODD, nCODD), we run them on the real sequence concatenated to its shuffled sequence, but only count the number of predicted domains on the shuffled portion of the sequence. Therefore, we count the random predictions that might have been rewarded by positive context not only with other random predictions, but also with potentially real domains from the real sequence (Figure $2 \mathrm{~A})$. The shuffling is performed 20 times for each sequence.

We estimate the false discovery rate (FDR) as follows. Let $A$ be the number of predictions per shuffled protein, and let $R$ be the number of predictions per real protein. Then $F D R=A / R$, which is a common approximation of the FDR [31]. To calculate the FDR of the new dPUC domains only, let $A_{n}$ and $R_{n}$ be the quantities defined above when we use negative context only, and $A$ and $R$ when we use the $E$-value threshold of interest for candidate domains. The difference of the data corresponds 


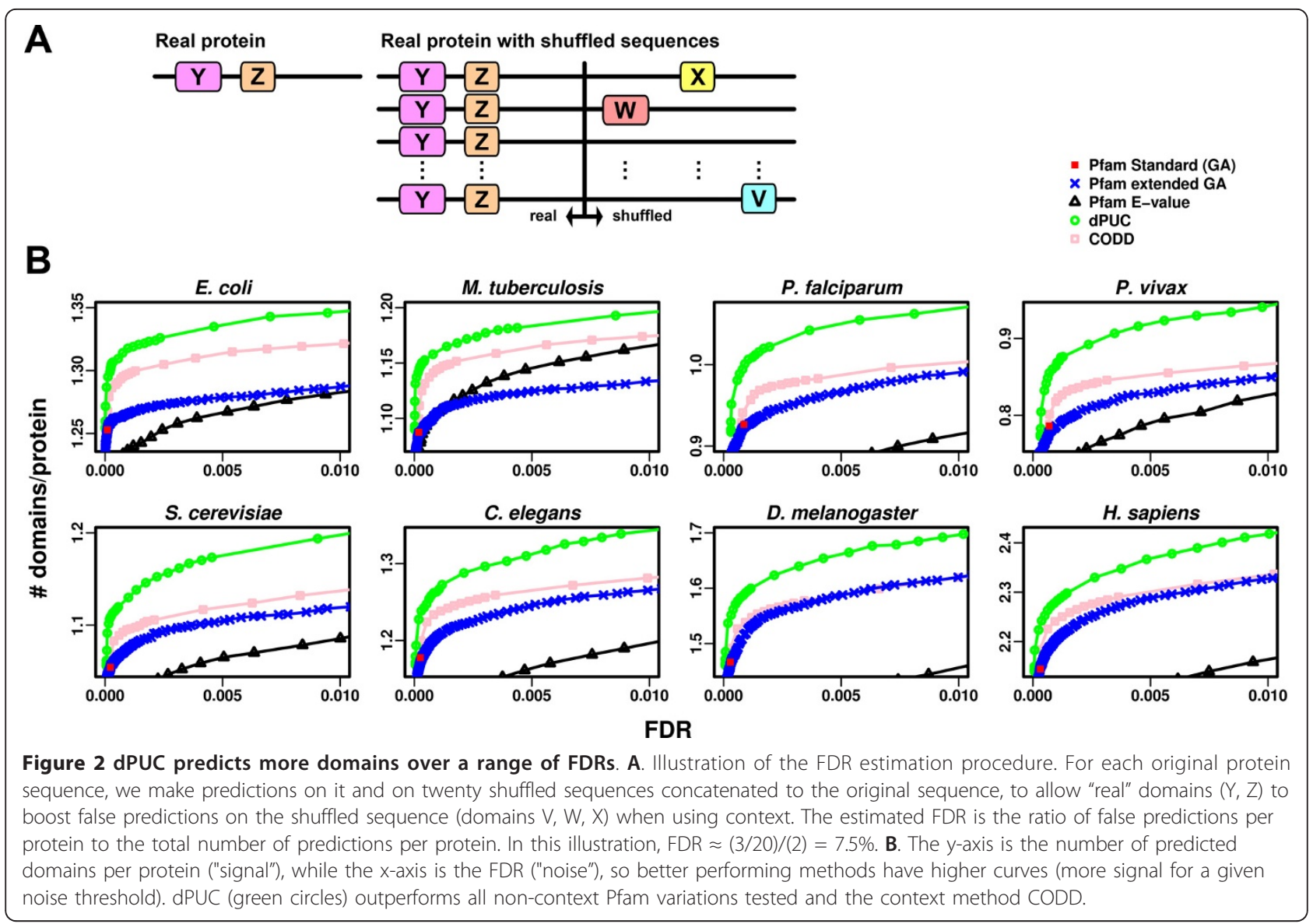

almost entirely to the novel domains, ignoring the effect of the initial negative context elimination:

$$
\text { FDR for new domains only } \approx\left(A-A_{n}\right) /\left(R-R_{n}\right) .
$$

This benchmark is only appropriate for methods in which the orientation of the domains is unimportant, as it is for the methods tested here. We note that the Markov model of Coin et al. [20] is sensitive to the orientation of domains, so its performance cannot be measured by this benchmark; we also note that an implementation of this program is not available online. See Additional File 1 "Estimated FDR details" for further details on estimating the FDR.

\section{Ortholog coherence scores}

In our second test, we measure how often domains are predicted across orthologs. The key assumption is that real domains are very likely to be present in orthologs, whereas false domains are very unlikely to be. Therefore, the average "ortholog coherence" score is inversely related to the amount of spurious predictions. We chose the Plasmodium species' proteins because their sequence divergence is large enough to discard "coherent" false predictions due to high sequence similarity, yet the sequences are similar enough for orthologs to be identified easily, and domain architectures are largely conserved. Moreover, as we show below, our approach performs well in improving domain identifications in these species.

We computed the orthologous groups of six Plasmodium species using OrthoMCL 1.4 [32]. We obtained 5582 orthologous groups ( 32,250 proteins). We eliminated orthologous groups with more than 13 proteins to avoid constructing large alignments and to ignore the well-studied large paralogous families that are characteristic of Plasmodium species (including PfEMP1, RIFIN, STEVOR, in P. falciparum and VIR, YIR, KIR, and CIR in the other species, which may bias our results). This left us with 5523 groups with a total of 30,065 proteins. Each group was aligned with T-Coffee 8.14 [33], using the M-Coffee special mode which combines the alignments of T-Coffee, ProbCons 1.12 [34], and Muscle 3.6 [35]. The score of a domain is the fraction of times we observed overlapping domains (after mapping to the alignment) of identical family in the orthologs. The score of a method is the average domain score over all proteins (Figure 3A). 


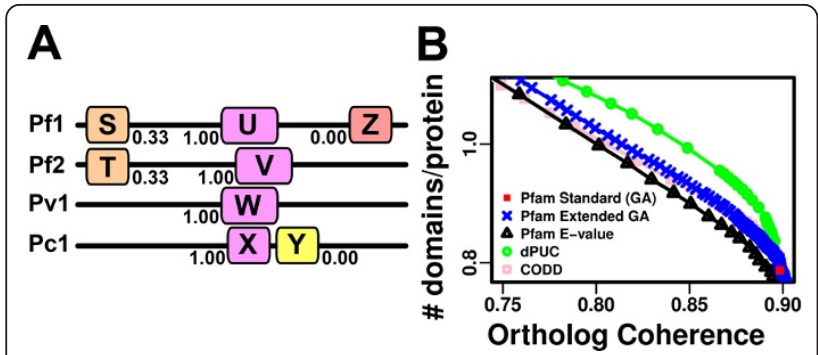

Figure 3 dPUC predicts more domains over a range of Ortholog Coherence scores on Plasmodium species. A. Illustration of scores. Domain predictions are made on hypothetical aligned orthologs and in-paralogs (Pf1, Pf2, Pv1, and PC1). Color denotes domain family. Domain $S$ overlaps $T$ of the same family, so their scores are 1/3 (since they lack predictions in Pv1 and PC1). In contrast, $U$ is predicted $100 \%$ in its orthologs and in-paralogs. $Y$ overlaps $V$ but is not of the same family, so its score is zero. Similarly, $Z$ does not overlap any domains. The score of this method is the average domain score on all proteins, $\sim 0.58$, while the average number of domains per protein is 2 . $\mathbf{B}$. The $y$-axis is the number of predicted domains per protein ("signal"), while the $x$-axis is the ortholog coherence score (inversely related with "noise"), so better performing methods have higher curves (more signal for a given noise threshold). dPUC (green circles) outperforms the other methods. Symbols and colors are as in Figure 2.

\section{Results}

We chose eight diverse and representative organisms to test our method, including human, four model organisms, D. melanogaster, C. elegans, S. cerevisiae, E. coli, and several pathogens including the eukaryotic human malaria parasites $P$. falciparum and $P$. vivax, and the prokaryotic parasite $M$. tuberculosis. We test our method separately on each of these organisms since they have different and sometimes extreme biases in protein lengths, amino acid compositions, amino acid coverage by domains, and domain family content (Additional File 1 Figure S2).

\section{dPUC improves Pfam predictions across all tested organisms and across a range of FDRs}

For a given method (e.g., Standard Pfam, dPUC, or CODD), we estimate its FDR as the ratio of the number of predictions made on shuffled sequences (when concatenated to real sequences, in the case of context methods), to the number of predictions made on the real sequences only. This concatenation approach allows noise in the shuffled sequence to be boosted by potentially real domains via context (Figure 2A). We test dPUC by varying the HMMER $E$-value threshold on its candidate domains. dPUC consistently enhances the performance of Standard Pfam across organisms and over the entire range of FDRs tested (Figure 2B). The Standard Pfam produces a single data point, and notably, its FDR is non-zero for all organisms. To explore Pfam's tradeoff between coverage and false positives, we vary the threshold to the HMMER $E$-value; this leads to fewer predictions on real sequences for the same FDR, showing that curation of Pfam thresholds has added value. We also vary the Pfam "gathering" thresholds by shifting them uniformly for all domain families, which performs better than $E$-value thresholds in all organisms except in $M$. tuberculosis, suggesting the Pfam curated domain thresholds are less appropriate for this diverged and compositionally biased (GC-rich) organism than they are for model organisms. Note that even when limiting the dPUC candidate domains to those predicted by the Standard Pfam (effectively when setting $E \leq 0.001$, the leftmost datapoint of the dPUC curves), we see a sharp decrease in dPUC's FDR relative to the Standard Pfam; this improved performance can be directly attributed to the removal of false predictions using negative context scores.

The FDR as described above applies to the entire set of predictions. For dPUC with low HMMER $E$-value thresholds on the candidate domains, the FDR is the net effect of removing domains from the Standard Pfam through negative context, as well as adding new domains with positive context. Since negative context alone reduces the FDR of the predictions, the FDR of the new domains must be larger than the FDR of the whole. For dPUC with $E \leq 1$, the FDR of the new domains only (see Methods) varies between $0.8-2 \%$, depending on the organism (see Additional File 1 Table S1).

\section{dPUC outperforms simple filters incorporating context}

We implemented an alternative context method, using filters, in which context scores are not defined. The first filter emulates the method CODD [24], in which candidate domains pass if they co-occurred with the domains that pass the Pfam gathering thresholds. Interestingly, the performance of CODD is similar to non-context methods in some organisms, and especially at high HMMER E-values thresholds (Figure 2B). We tested CODD using the published CODD positive context network, which notably lacks context between domains of the same family, and additionally removes observed domain pairs that do not occur more often than expected from the hypergeometric distribution. We note, however, that using the CODD filter with the dPUC network of positive context domain pairs improves the predictions compared to using this more limited CODD network (Additional File 1 Figure S3). Our previous analysis suggested that a negative filter (to mirror our negative scores) was necessary to enhance the FDR, and indeed, our approach of a double positive and negative filter, nCODD (see Additional File 1 Supplementary Methods), has better performance than CODD (Additional File 1 Figure S3). Nevertheless, nCODD falls behind dPUC in all organisms tested, with 
the exception of $D$. melanogaster and $H$. sapiens, in which performance is similar to dPUC's; this could be due to the overall better annotations of these two model organisms. In P. falciparum, nCODD is too aggressive and removes predictions from the real sequences as well as from the shuffled sequences, shifting the entire curve downward relative to dPUC. These results suggest that there is added value in our setup of scores and thresholds, compared to these simpler filter formulations.

\section{Performance of chosen context scores}

Our context scores have a specific form (log-odds, see Methods) in which a pair score is logarithmically proportional to how often the domain family pair is observed in Standard Pfam. Although these log-odds scores seem naturally compatible with the HMMER logodds scores, it is not certain that this is the form the scores should have. To test the importance of these scores, we shuffled the positive scores (three times), which resulted in decreased performance compared to our original context scores, but better performance than that of non-context methods (data not shown). Therefore, carefully choosing values for our scores is important, and our form, which weighs the evidence of cooccurrence per domain family pair, performs better than random scores from the same distribution. Additional score parameter variations are discussed in Additional File 1 "dPUC Pfam parameter robustness".

\section{dPUC increases Pfam coverage}

We choose a threshold of $E \leq 1$ to identify the candidate domains, which corresponds to an overall FDR of $0.03-0.2 \%$ across organisms; that is, this is within an order of magnitude of the Standard Pfam FDR of 0.010.09\% (Additional File 1 Table S1). We then calculate the dPUC net percent improvements compared to the Standard Pfam (Table 1). The number of domains

Table 1 dPUC increases domain predictions and amino acid coverage

\begin{tabular}{|c|c|c|c|c|c|c|c|c|}
\hline & E. c. & M. $t$. & P. f. & P. v. & S. c. & C. e. & $\begin{array}{l}D . \\
m .\end{array}$ & H. s. \\
\hline Domains & 4.30 & 6.00 & 10.30 & 11.46 & 6.21 & 8.07 & 9.08 & 7.15 \\
\hline $\begin{array}{l}\text { Domains unique } \\
\text { families }\end{array}$ & 2.62 & 2.91 & 5.73 & 5.60 & 3.38 & 3.66 & 4.38 & 3.43 \\
\hline $\begin{array}{l}\text { Domains } \\
\text { repeated } \\
\text { families }\end{array}$ & 26.56 & 39.61 & 23.83 & 37.48 & 23.79 & 19.95 & 18.26 & 12.34 \\
\hline Amino acids & 2.38 & 4.13 & 7.25 & 7.66 & 3.14 & 4.74 & 5.63 & 3.63 \\
\hline Proteins & 0.16 & 0.08 & 1.80 & 1.31 & 0.38 & 0.70 & 0.59 & 0.56 \\
\hline
\end{tabular}

Percent increases of dPUC predictions relative to the Standard Pfam are given for each organism (E. C., E. coli; M. t., M. tuberculosis; P. f., P. falciparum; P. v., P. vivax; S. C., S. cerevisiae; C. e., C. elegans; D. m., D. melanogaster; $H$. s., $H$. sapiens) when considering all domains, first appearance of domains in a protein, subsequent occurrences of domains in a protein, amino acids covered by a domain, and all proteins with domain predictions. (See text.) increases by $4-11 \%$, with a trend roughly inverse to the level of initial coverage in each organism (Additional File 1 Figure S2D). Unique domain families increase by $3-6 \%$, while repeated families increase at the higher rates of $12-40 \%$. Amino acid coverage improves by $2-8 \%$ relative to the amino acids covered by the Standard Pfam. However, most new predictions appear in proteins that have Standard Pfam domains, since there are smaller increases in protein coverage (0.1-1.8\%). Overall, the two Plasmodium species attain the highest increases in coverage, but even the best-annotated model organism, $E$. coli, experiences increases in coverage under all metrics (Table 1).

\section{dPUC leads to additional functional annotations}

We used the MultiPfam2GO procedure [3] to obtain $\mathrm{GO}$ annotations from our domain predictions. This program uses a probabilistic approach to determine how sets of domains imply GO terms. We ran this procedure on the Standard Pfam and dPUC predictions, and filtered the results so that only the most specific GO terms remained (by removing all ancestors using all GO relationships).

In total, $2.5-7.8 \%$ of proteins had new or modified GO terms, depending on the organism. As we observed before with Pfam coverage, the two Plasmodium species attained the largest increases in GO terms. We present the summary of our data in Table 2 (detailed counts are in Additional File 1 Table S2). The vast majority of the original GO terms from Standard Pfam (over 97\%) are preserved by dPUC with $E \leq 1$. Additionally, we obtain a $1.2-4.0 \%$ increase in $\mathrm{GO}$ terms that are completely new, and a further $0.4-0.9 \%$ in GO terms that are more specific than previously existing GO terms. Our procedure also results in a negligible $0.2-0.7 \%$ of GO terms in dPUC becoming less specific than their Standard Pfam counterparts, and $0.3-1.4 \%$ GO terms being deleted. Similarly, most proteins with GO terms from Standard Pfam are unchanged by dPUC, but $1.8-5.0 \%$ of proteins

Table 2 dPUC predictions lead to novel or more specific Gene Ontology terms on proteins

\begin{tabular}{lrrrrrrrr}
\hline & E. c. & M. t. & P. f. & P. v. & S. c. & C. e. & $\begin{array}{r}\text { D. } \\
\text { m. }\end{array}$ & H. s. \\
\hline Same & 98.51 & 98.27 & 96.86 & 96.01 & 98.28 & 97.07 & 96.26 & 96.68 \\
$\begin{array}{l}\text { New or more } \\
\text { specific }\end{array}$ & 1.80 & 1.98 & 5.05 & 5.00 & 2.04 & 3.07 & 3.40 & 3.13 \\
$\begin{array}{l}\text { Deleted or less } \\
\text { specific }\end{array}$ & 0.39 & 0.52 & 0.88 & 1.25 & 0.32 & 0.90 & 1.12 & 0.86 \\
Mixed & 0.28 & 0.49 & 1.07 & 1.53 & 0.72 & 1.17 & 1.61 & 1.36
\end{tabular}

Comparison of dPUC-based GO predictions with those based on the Standard Pfam. Values are percents relative to the number of proteins with $\mathrm{GO}$ terms in the Standard Pfam per organism. Each category is mutually exclusive, with "Mixed" specifying that both "new or more specific" and "deleted or less specific" GO terms occurred in the same proteins. 
have new or more specific GO terms, while only 0.3 $1.3 \%$ of proteins have fewer or less specific GO terms. Finally, $0.3-1.5 \%$ of proteins experience both increased and decreased specificity of GO terms.

As expected, inspection reveals that most GO terms that are deleted or become less specific are a consequence of either domain replacement brought upon by positive context (which are usually accompanied by new GO terms) or domain removal due to negative context. In either case, we expect the new domain predictions to be more accurate, and the resulting removal of GO terms is welcomed. Interestingly, the addition of domains can also lead to GO term loss, which is a consequence of the MultiPfam2GO probabilistic model combined with incomplete training data (a detailed example is presented in Additional File 1 "Novel domain predictions may lead to GO term deletions with MultiPfam2GO”).

\section{Domain coherence is enhanced across Plasmodium orthologs}

We chose to focus on the Plasmodium parasites, the causative agents of malaria, due to their wide impact on human health, and also because our method showed the largest improvements in these organisms. A simple test of prediction quality is to ask if domains are predicted in orthologous sequences as well, since orthology information is not exploited by our method. Importantly, ortholog co-prediction is expected to be low for false predictions and high for real domains, providing us with an alternative measure of noise that does not depend on statistical simulations.

We defined an "ortholog coherence" score between 0 and 1, namely, the average fraction of times a domain is predicted in orthologous proteins (Figure 3A). We looked at the proteins of six Plasmodium species (P. falciparum, P. vivax, P. knowlesi, P. chabaudi, P. berghei, and $P$. yoelii) with orthologs or in-paralogs in these organisms as predicted by OrthoMCL, and plotted these scores against their number of domain predictions per protein for the same methods tested earlier (Figure 3B, Additional File 1 Figure S4). These coherence scores may be artificially low due to artefacts in the alignments or gene models (that is, exons might be missing in some orthologs), but on average all methods should be affected equally. Using dPUC with $\mathrm{E} \leq 1$ increases domain predictions by $11 \%$ at practically the same ortholog coherence (a $0.87 \%$ decrease) as the Standard Pfam. Coherence scores recapitulate our conclusions derived independently from our FDR analysis, displaying an increase in domain predictions over a wide range of ortholog coherence thresholds.

\section{Novel $P$. falciparum annotations}

Careful manual analysis of novel domain predictions on the $P$. falciparum proteome led to the reannotation of
55 proteins, either due to novel Pfam domains that had not been predicted before (Table 3 ) or due to novel Pfam domains confirmed by other domain databases (Table 4). Our discoveries include the identification of orthologs to many core machinery proteins conserved in all eukaryotes, including those involved in ribosomal assembly and other RNA processing events, which surprisingly had not been previously known (full details in the Additional File 1 "New annotations on P. falciparum"). Three predictions find direct support in the literature. PF11_0086, the predicted "poly(A)-binding protein-interacting protein 1" PAIP1, has a strong yeast 2-hybrid interaction with PFL1170w [36], the annotated "poly(A)-binding protein" PABP1 homolog in P. falciparum. Additionally, the two DEAD-box helicases PFE1390w and MAL8P1.19, predicted to be the orthologs of ABSTRAKT and DBP10 respectively, agree with the assignments of a recently published curated list of $P$. falciparum helicases [37]. Such new functional annotations can serve as starting points for developing new therapeutic intervention strategies. Our suggestions have been submitted to PlasmoDB [28] as community annotations.

\section{Discussion}

We have shown that domain identification can be greatly improved across a diverse set of organisms by exploiting domain co-occurrence information. Our method is more successful at increasing domains in genomes with lower domain coverage (Table 1 and Additional File 1 Figure S2D) and shows greatest improvements in the least annotated of the organisms we tested, the malaria parasites.

To our knowledge, there has been limited prior work examining the utility and systematic use of domain context for domain prediction [20], and to date such approaches have not been widely adopted, nor are they publicly available. Coin et al. developed a Markov model framework to score the dependence between domains in a sequence. However, their work presents two versions of the Markov model, each of which has opposing limitations: the first-order Markov model has few parameters, but its predictive power is limited; alternatively, their $k^{\text {th }}$-order Markov model has more power $(k=5$ gives twice as many predictions as $k=1$ ), at the cost of an exponential (in $k$ ) increase in parameters, which become difficult to estimate accurately and increase the computational requirements of the approach. In contrast, dPUC allows interactions between all domains, as opposed to just the previous $k$ domains, while keeping the parameter space small, simplifying parameter estimation and reducing our program's memory usage. Furthermore, the source code accompanying our work 
Table 3 Completely novel P. falciparum dPUC predictions lead to refined protein annotations

\begin{tabular}{|c|c|c|c|c|}
\hline Protein ID & $\begin{array}{l}\text { Standard } \\
\text { Pfam } \\
\text { domains }\end{array}$ & $\begin{array}{l}\text { Additional } \\
\text { dPUC } \\
\text { domains }\end{array}$ & $\begin{array}{l}\text { Current annotation } \\
\text { (PlasmoDB 6.0) }\end{array}$ & Suggested reannotation (this study) \\
\hline PFL0980w & CwfJ_C_1 & CwfJ_C_2 & $\begin{array}{l}\text { conserved Plasmodium } \\
\text { protein, unknown function }\end{array}$ & $\begin{array}{l}\text { Debranching enzyme-associated ribonuclease (DRN1 ortholog), } \\
\text { putative }\end{array}$ \\
\hline PF13_0222 & Metallophos & DBR1 & phosphatase, putative & RNA lariat debranching enzyme (DBR1 ortholog), putative \\
\hline PF11_0086 & MIF4G & PAM2 & $\begin{array}{l}\text { MIF } 4 \text { G domain containing } \\
\text { protein }\end{array}$ & $\begin{array}{l}\text { Poly(A)-binding protein-interacting protein } 1 \text { (PAIP1 ortholog), } \\
\text { putative }\end{array}$ \\
\hline PFE1390w & $\begin{array}{l}\text { DEAD, } \\
\text { Helicase_C }\end{array}$ & zf-CCHC & RNA helicase-1 & Post-translational mRNA regulation (ABSTRAKT ortholog), putative \\
\hline PF08_0130 & WD40 & Utp13 & WD-repeat protein, putative & U3 ribonucleoprotein component (PWP2 ortholog), putative \\
\hline PF14_0456 & WD40 & Utp12 & $\begin{array}{l}\text { conserved Plasmodium } \\
\text { protein, unknown function }\end{array}$ & U3 ribonucleoprotein component (DIP2 ortholog), putative \\
\hline PF10_0128 & WD40 & Utp13 & WD-repeat protein, putative & U3 ribonucleoprotein component (UTP13 ortholog), putative \\
\hline PFI1025w & RRM_1 & Lsm_interact & $\begin{array}{l}\text { RNA binding protein, } \\
\text { putative }\end{array}$ & U4/U6 snRNA-associated-splicing factor (PRP24 ortholog), putative \\
\hline PFL0985c & DUF367 & RLI & $\begin{array}{l}\text { conserved protein, } \\
\text { unknown function }\end{array}$ & Ribosome biogenesis regulator (TSR3 ortholog), putative \\
\hline MAL8P1.19 & $\begin{array}{l}\text { DEAD, } \\
\text { Helicase_C }\end{array}$ & DBP10CT & RNA helicase, putative & Ribosomal biogenesis RNA helicase protein (DBP10 ortholog), putative \\
\hline PFE0560c & MORN & Avl9 & $\begin{array}{l}\text { MORN repeat protein, } \\
\text { putative }\end{array}$ & $\begin{array}{l}\text { Atypical Golgi transport protein (AVL9 ortholog) with MORN domains, } \\
\text { putative }\end{array}$ \\
\hline PFL1455W & DUF202, SPX & VTC & $\begin{array}{l}\text { conserved Plasmodium } \\
\text { protein, unknown function }\end{array}$ & Vacuolar transporter chaperone (VTC2/3/4 ortholog), putative \\
\hline PFL2255W & TPR_2 & F-box & $\begin{array}{l}\text { conserved Plasmodium } \\
\text { protein, unknown function }\end{array}$ & DNA replication origin binding protein (DIA2 ortholog), putative \\
\hline PFF1070c & $\begin{array}{l}\text { UPF0004, } \\
\text { Radical_SAM }\end{array}$ & TRAM & $\begin{array}{l}\text { radical SAM protein, } \\
\text { putative }\end{array}$ & $\begin{array}{l}\text { Ribosome or tRNA methylthiotransferase (RIMO or MIAB ortholog) or } \\
\text { CDK5 regulatory subunit-associated protein 1, putative }\end{array}$ \\
\hline PFL1045w & DUF814 & FbpA & $\begin{array}{l}\text { conserved protein, } \\
\text { unknown function }\end{array}$ & FbpA domain protein, putative \\
\hline MAL13P1.182 & RanBPM_CRA & $\mathrm{LisH}$ & $\begin{array}{l}\text { conserved Plasmodium } \\
\text { protein, unknown function }\end{array}$ & GID8 ortholog, putative \\
\hline MAL13P1.79 & & zf-CCCH, WD40 & $\begin{array}{l}\text { conserved Plasmodium } \\
\text { protein, unknown function }\end{array}$ & $\mathrm{CCCH}$ zinc finger protein, putative \\
\hline MAL13P1.37 & & zf-B_box & $\begin{array}{l}\text { conserved Plasmodium } \\
\text { protein, unknown function }\end{array}$ & Tripartite motif protein, putative \\
\hline
\end{tabular}

These dPUC domain predictions were novel relative to the Standard Pfam, SMART, and Superfamily, and coherent predictions were present in OrthoMCL orthologs or in-paralogs. dPUC predictions always contained the Standard Pfam domains, so only the additional domain predictions are listed. The number of repeats per family is not shown.

should facilitate further improvements in context-based identification of domains.

Recently, a method was published (CODD) that uses a list of "favored" domain pairs to predict lower-scoring domains in P. falciparum if high scoring domains that preferentially appear with them are found in the same sequence [24]. We have shown that dPUC significantly outperforms such a filter (Figure 2 and Figure 3), as it predicts many more domains at any fixed FDR. There are several important methodological contributions of dPUC that together explain this performance enhancement. First, our method penalizes (but does not necessarily eliminate) domain pairs that have never been observed before, whereas CODD uses only information about favored domain pairs. Second, our method uses domain family pair-specific log-odds scores and thresholds, while CODD treats all favored domain family pairs equally. Third, our method allows weak domains to boost each other in the absence of strong domains without any special treatment, while CODD has to be run separately to allow candidate domains to predict each other, and with different $E$-value thresholds since this is much more prone to false predictions. Fourth, our problem is combinatorial in nature, allowing domains with low $E$-values to overcome higher $E$-value candidates if the context is more favorable, while CODD simply prioritizes candidate domains by $E$-value. Fifth, CODD uses a limited positive context network that does not include repeating domains and eliminates pairs that do not have small enough p-values derived from the hypergeometric distribution. Although our benchmarks show that negative context and our more complete dPUC positive context networks are critical differences (Additional File 1 Figure S3 and Figure S4), these features 
Table 4 Additional P. falciparum dPUC predictions lead to refined protein annotations

\begin{tabular}{|c|c|c|c|}
\hline Protein ID & $\begin{array}{l}\text { Standard Pfam } \\
\text { domains }\end{array}$ & $\begin{array}{l}\text { Additional dPUC } \\
\text { domains }\end{array}$ & Suggested reannotation (this study) \\
\hline PFE1240w & $\begin{array}{l}\text { Radical_SAM, } \\
\text { Wyosine_form }\end{array}$ & Flavodoxin_1 & Wybutosine synthesis protein (TYW1 ortholog), putative \\
\hline PFF1490w & THF_DHG_CYH_C & THF_DHG_CYH & $\begin{array}{l}\text { Tetrahydrofolate dehydrogenase/cyclohydrolase (MTD1 ortholog, MIS1/ADE3 homolog } \\
\text { without FTHFS domain), putative }\end{array}$ \\
\hline MAL8P1.139 & $\mathrm{DDA} 1^{*}$ & WD40 & Regulator of $(\mathrm{H}+$ )-ATPase in Vacuolar membrane (RAV1 ortholog), putative \\
\hline PF08_0124 & CactinC_cactus & Cactin_mid & CACTIN homolog, putative \\
\hline PF10_0152 & & $\begin{array}{l}\text { NTP_transf_2, } \\
\text { PAP_assoc }\end{array}$ & $\begin{array}{l}\text { Non-canonical cytoplasmic specific poly(A) RNA polymerase protein (CID13 ortholog), } \\
\text { putative }\end{array}$ \\
\hline MAL13P1.170 & NTP_transf_2 & PAP_assoc & Non-canonical poly(A) RNA polymerase protein (PAP2/TRF5 ortholog), putative \\
\hline PFI1560c & DUF21 & CBS, CNMP_binding & Required for mitochondrial morphology (MAM3 ortholog), putative \\
\hline PF10_0126 & & WD40 & Phosphoinositide binding protein (HSV2/ATG18 ortholog), putative \\
\hline PFI0510c & $\mathrm{BRCT}$ & IMS & DNA repair protein (REV1 ortholog), putative \\
\hline MAL13P1.54 & WD40 & $\mathrm{LisH}$ & Alternative splicing regulator (SMU-1 ortholog), putative \\
\hline PF14_0052 & cobW & CobW_C & COBW domain-containing protein 1 (CBWD1 ortholog), putative \\
\hline PF08_0012 & SET, Pre-SET & YDG_SRA & Histone lysine N-methyltransferase, putative \\
\hline PFE1445c & & FG-GAP & T-cell immunomodulatory protein (human TIP homolog), putative \\
\hline PFL0975w & IQ & $\mathrm{RCC1}$ & Unconventional myosin fused to IQ and RCC1 domains, putative \\
\hline PF11_0276 & Abhydro_lipase & Abhydrolase_1 & Steryl ester hydrolase (TGL1/YEH1/YEH2 ortholog), putative \\
\hline PF13_0190 & Aha1_N & TPR_2, TPR_1 & Chaperone binding protein, putative \\
\hline PF11_0287 & CRAL_TRIO & CRAL_TRIO_N & CRAL/TRIO protein, putative \\
\hline PF11_0197 & Ank & ACBP & Acyl-CoA-binding protein, putative \\
\hline PF14_0647 & TLD & TBC & Rab GTPase activator, putative \\
\hline PFL0575w & $\begin{array}{l}\text { Amino_oxidase, } \\
\text { Thi4* }\end{array}$ & PHD & PHD finger and flavin containing amine oxidoreductase, putative \\
\hline MAL13P1.246 & E1-E2_ATPase & Cation_ATPase_C & E1-E2 ATPase, putative \\
\hline PF11_0116 & & Nol1_Nop2_Fmu & Nol1/Nop2/Fmu-like protein, putative \\
\hline MAL7P1.127 & & Pkinase & Rab GTPase activator and protein kinase, putative \\
\hline PFC0425w & & zf-C3HC4, PHD & PHD finger protein, putative \\
\hline PFI0975C & & $\mathrm{RCC1}$ & Regulator of chromosome condensation, putative \\
\hline PFD0900w & & RCC1 & Regulator of chromosome condensation, putative \\
\hline MAL7P1.132 & & Pkinase & Protein kinase, putative \\
\hline PFF0810c & & Ras & Ras GTPase, putative \\
\hline PFL1990c & & zf-CCHC, RRM_1 & RNA binding protein, putative \\
\hline PF07_0066 & & RRM_1 & RNA binding protein, putative \\
\hline PF13_0147 & & RRM_1 & RNA binding protein, putative \\
\hline PFF1120c & & EGF & EGF-like membrane protein, putative \\
\hline PF14_0262 & WD40 & TPR_1 & WD40 and TPR repeats protein, putative \\
\hline PFI0275w & & WD40 & WD40 repeat and EF hand protein, putative \\
\hline PF10_0285 & & WD40 & WD40 repeat protein, putative \\
\hline PF11_0195 & & WD40 & WD40 repeat protein, putative \\
\hline PF14_0640 & & WD40 & WD40 repeat protein, putative \\
\hline MAL13P1.308 & & Arm & ARM repeat protein, putative \\
\hline
\end{tabular}

These dPUC predictions were novel compared to the Standard Pfam, and were consistent with existing domain predictions from SMART or Superfamily (and often present in orthologs too). The number of repeats per family is not shown.

dPUC predictions always contained the Standard Pfam domains, so only the additional domains are listed, except when marked with an asterisk ${ }^{*}$; MAL8P1.139 has DDA1 in Standard Pfam but not in dPUC Pfam; PFL0575w has a Thi4 in Standard Pfam but it is replaced by another Amino_oxidase domain [belonging to the same Pfam clan] in dPUC Pfam).

All proteins have the current PlasmoDB 6.0 annotation of "conserved Plasmodium protein, unknown function" except: MAL8P1.139, PFI1560c, MAL13P1.246, MAL7P1.127 "conserved Plasmodium membrane protein, unknown function"; PFE1240w, PF11_0287 "conserved protein, unknown function"; MAL13P1.170 "nucleotidyltransferase, putative"; PF08_0012 "SET domain protein, putative"; PFF1120c "conserved Apicomplexan protein, unknown function"; PF14_0262 "probable protein, unknown function". 
alone do not explain dPUC's higher performance, suggesting that the other differences also play an important role. The importance of negative context is consistent with the fact that domain context is usually not transitive. In particular, we find that if a Pfam domain A cooccurs with domains $\mathrm{B}$ in at least one sequence in Uniprot and it co-occurs with domain $\mathrm{C}$ in at least one sequence, then domains $\mathrm{B}$ and $\mathrm{C}$ co-occur in at least one sequence only $15.7 \%$ of the time; however, domain pairs that do not co-occur can readily be predicted by methods that only reward favored domain pairs.

Other works, such as AIDAN [38] and a similar work [39], have also used domain context to refine domain predictions in a manner complementary to the work discussed here. In particular, while our method learns parameters from domain databases and uses these to make predictions on each protein sequence independently, AIDAN clusters sequences based on the original domain predictions, and refines these predictions by performing domain architecture alignments and evaluating sequence comparisons within these clusters when domains are missing or are mismatched. Further, the thresholds used by AIDAN are tuned so less than $0.05 \%$ of the new domain assignments are errors. The AIDAN approach can be used as a second step in any domain prediction pipeline, including our own, to further refine predictions by directly taking the sequence similarity of related proteins into account, and this is a promising avenue for future work.

Domain context is independent of the information that HMM scores capture, since an HMM score only uses the sequence the domain encompasses, ignoring the surrounding sequence that contains the rest of the domains, while domain context allows these other domains to affect each other. These two information sources do not overlap. In other words, HMMs capture so-called "vertical" information [40] while domain context captures "horizontal" information. This is similar to advances in remote homology detection due to the incorporation of secondary structure predictions in addition to HMM/profile information [40-42]. Fuzzy HMMs [43] have been proposed to model positional dependence within a domain, but currently these predictions are less tractable compared to classical HMM algorithms. We believe that incorporating horizontal information in HMM analysis will become more common, as it is evidently an important means for boosting subtle sequence signals.

We have implemented a log-odds system to score the co-occurrence of domain pairs. Indeed, log-odds scores are widespread in bioinformatics, and our scoring system is inspired by the log-odds scores used in local sequence alignment (LSA) [44]. We borrowed the concept that optimal scores ought to be log ratios of the "target" frequencies versus the "background" frequencies. Broadly speaking, there are many similarities in both problems, including that they score pairs and that the searches are both "local" (LSA seeks the best subsequences, while dPUC seeks the best subset of domains). However, an important assumption of the theory behind LSA is that different amino acid pairs are uncorrelated, whereas domain pair scores in APUC are correlated, since solutions must be connected as a full clique (see the solution in Figure 1B). Our framework does not handle these correlations, and while we have demonstrated empirically that log-odds scores perform remarkably well, we expect that a better theoretical foundation for our problem (which might explicitly relate arbitrary scores to statistical parameters) will lead to scores that yield better performance. Other changes in the dPUC scoring system may also lead to improvements; for example, differentiating between the two orderings of domain pairs is a promising avenue for future research, as it has been shown that many domain pairs occur in a single order [18].

While we applied dPUC to Pfam using HMMER2, there are other tools available which could be used, including SAM [45] and the new HMMER3 [21], as well as other domain databases, like SMART, Superfamily, and CDD. More broadly, the dPUC framework could be used to improve prediction of other protein sequence features, including signal sequences and transmembrane domains, both of which co-occur with a limited number of domains. The same underlying mathematical framework may also be applicable in other diverse settings, for example, in uncovering cis-regulatory modules, as transcription factors often work cooperatively to regulate genes. We have shown that dPUC significantly improves predictions over other models that score domains independently. We anticipate that our framework will be useful for incorporating context with other DNA, RNA, or protein sequence features and will find its greatest utility in the annotation of newly sequenced genomes from highly diverged organisms.

\section{Conclusions}

Common domain identification methods typically consider each domain separately, and they have limited applicability when the similarity between the query sequence and known domains is very low, as is the case for poorly understood genomes. Here we significantly improved domain predictions by exploiting the tendency for domains to co-occur in specific combinations. We developed an approach based on the observation that weak domain predictions are better supported if they appear in previously-observed combinations, while domain combinations that have never been observed are less likely to be valid. Our method improved domain 
predictions in all organisms tested, including the best known model organisms. The biggest improvements were seen for the divergent organism Plasmodium falciparum, the parasitic agent of malaria, for which much of the core cellular machinery remains unidentified. Overall, our approach is likely to be most useful for poorly understood genomes where the need for additional annotations is arguably the greatest.

\section{Additional material}

Additional file 1: Supplementary information. PDF file (17 pages) that includes all the supplementary methods, results, figures and tables.

\section{Acknowledgements}

We thank all members of the Singh and Llinás groups for helpful discussions about this work. We additionally thank Tao Yue, Erandi De Silva, Hani Goodarzi, and our reviewers for their feedback on this manuscript. This work was supported by the National Science Foundation [Graduate Research Fellowship DGE 0646086 to AO]; and the National Institutes of Health [1 R21-Al085415 to MS and ML, Center of Excellence P50 GM071508 to the Lewis-Sigler Institute].

\section{Author details}

'Department of Molecular Biology, Princeton University, Princeton, USA. ${ }^{2}$ Department of Computer Science, Princeton University, Princeton, USA. ${ }^{3}$ Lewis-Sigler Institute for Integrative Genomics, Princeton University, Princeton, USA.

\section{Authors' contributions}

$\mathrm{AO}$ and $\mathrm{MS}$ conceived and designed the approach and experiments. $\mathrm{AO}$ implemented the approach and performed the experiments. All authors analyzed the data, wrote, read and approved the final manuscript.

Received: 25 October 2010 Accepted: 31 March 2011

Published: 31 March 2011

\section{References}

1. Stein L: Genome annotation: from sequence to biology. Nat Rev Genet 2001, 2:493-503.

2. Schug J, Diskin S, Mazzarelli J, Brunk BP, Stoeckert CJ: Predicting Gene Ontology Functions from ProDom and CDD Protein Domains. Genome Res 2002, 12:648-655.

3. Forslund K, Sonnhammer ELL: Predicting protein function from domain content. Bioinformatics 2008, 24:1681-1687.

4. Wilson D, Pethica R, Zhou Y, Talbot C, Vogel C, Madera M, Chothia C, Gough J: SUPERFAMILY-sophisticated comparative genomics, data mining, visualization and phylogeny. Nucl Acids Res 2009, 37:D380-386.

5. Marchler-Bauer A, Anderson JB, Chitsaz F, Derbyshire MK, DeWeese-Scott C, Fong JH, Geer LY, Geer RC, Gonzales NR, Gwadz M, He S, Hurwitz DI, Jackson JD, Ke Z, Lanczycki CJ, Liebert CA, Liu C, Lu F, Lu S, Marchler GH, Mullokandov M, Song JS, Tasneem A, Thanki N, Yamashita RA, Zhang D, Zhang N, Bryant SH: CDD: specific functional annotation with the Conserved Domain Database. Nucl Acids Res 2009, 37:D205-210.

6. Ye Y, Godzik A: Comparative Analysis of Protein Domain Organization. Genome Res 2004, 14:343-353.

7. Coulson RMR, Hall N, Ouzounis C: Comparative Genomics of Transcriptional Control in the Human Malaria Parasite Plasmodium falciparum. Genome Res 2004, 14:1548-1554.

8. Iyer LM, Anantharaman V, Wolf MY, Aravind L: Comparative genomics of transcription factors and chromatin proteins in parasitic protists and other eukaryotes. Int J Parasitol 2008, 38:1-31.

9. Przytycka T, Davis G, Song N, Durand D: Graph Theoretical Insights into Dollo Parsimony and Evolution of Multidomain Proteins. J Comput Biol 2006, 13:351-363.
10. Fong JH, Geer LY, Panchenko AR, Bryant SH: Modeling the Evolution of Protein Domain Architectures Using Maximum Parsimony. J Mol Biol 2007, 366:307-315.

11. Weiner J, Moore A, Bornberg-Bauer E: Just how versatile are domains? BMC Evol Biol 2008, 8:285.

12. Sprinzak E, Margalit $\mathrm{H}$ : Correlated sequence-signatures as markers of protein-protein interaction. J Mol Biol 2001, 311:681-692.

13. Deng M, Mehta S, Sun F, Chen T: Inferring Domain-Domain Interactions From Protein-Protein Interactions. Genome Res 2002, 12:1540-1548.

14. Guimarães K, Jothi R, Zotenko E, Przytycka T: Predicting domain-domain interactions using a parsimony approach. Genome Biol 2006, 7:R104.

15. Kanaan SP, Huang C, Wuchty S, Chen DZ, Izaguirre JA: Inferring ProteinProtein Interactions from Multiple Protein Domain Combinations. Method Mol Biol 2009, 541:43-59.

16. Song N, Joseph JM, Davis GB, Durand D: Sequence Similarity Network Reveals Common Ancestry of Multidomain Proteins. PLoS Comput Biol 2008, 4:e1000063.

17. Liu J, Rost B: CHOP: parsing proteins into structural domains. Nucl Acids Res 2004, 32:W569-W571.

18. Vogel C, Berzuini C, Bashton M, Gough J, Teichmann SA: Supra-domains: Evolutionary Units Larger than Single Protein Domains. J Mol Biol 2004, 336:809-823.

19. Moore AD, Björklund ÅK, Ekman D, Bornberg-Bauer E, Elofsson A: Arrangements in the modular evolution of proteins. Trends Biochem Sci 2008, 33:444-451.

20. Coin L, Bateman A, Durbin R: Enhanced protein domain discovery by using language modeling techniques from speech recognition. $P$ Nat Acad Sci USA 2003, 100:4516-4520.

21. Finn RD, Mistry J, Tate J, Coggill P, Heger A, Pollington JE, Gavin OL, Gunasekaran P, Ceric G, Forslund K, Holm L, Sonnhammer ELL, Eddy SR, Bateman A: The Pfam protein families database. Nucl Acids Res 2010, 38 D211-222.

22. Krogh A, Brown M, Mian IS, Sjölander K, Haussler D: Hidden Markov Models in Computational Biology: Applications to Protein Modeling. J Mol Biol 1994, 235:1501-1531.

23. Letunic I, Doerks T, Bork P: SMART 6: recent updates and new developments. Nucl Acids Res 2009, 37:D229-232.

24. Terrapon N, Gascuel O, Marechal E, Brehelin L: Detection of new protein domains using co-occurrence: application to Plasmodium falciparum. Bioinformatics 2009, 25:3077-3083.

25. The UniProt Consortium: The Universal Protein Resource (UniProt) 2009. Nucl Acids Res 2009, 37:D169-174

26. Gardner MJ, Hall N, Fung E, White O, Berriman M, Hyman RW, Carlton JM, Pain A, Nelson KE, Bowman S, Paulsen IT, James K, Eisen JA, Rutherford K, Salzberg SL, Craig A, Kyes S, Chan MS, Nene V, Shallom SJ, Suh B, Peterson J, Angiuoli S, Pertea M, Allen J, Selengut J, Haft D, Mather MW, Vaidya AB, Martin DMA, Fairlamb AH, Fraunholz MJ, Roos DS, Ralph SA, McFadden Gl, Cummings LM, Subramanian GM, Mungall C, Venter JC, Carucci DJ, Hoffman SL, Newbold C, Davis RW, Fraser CM, Barrell B: Genome sequence of the human malaria parasite Plasmodium falciparum. Nature 2002, 419:498-511.

27. Carlton JM, Adams JH, Silva JC, Bidwell SL, Lorenzi H, Caler E, Crabtree Angiuoli SV, Merino EF, Amedeo P, Cheng Q, Coulson RMR, Crabb BS, del Portillo HA, Essien K, Feldblyum TV, Fernandez-Becerra C, Gilson PR, Gueye AH, Guo X, Kang/'a S, Kooij TWA, Korsinczky M, Meyer EV-S, Nene V, Paulsen I, White O, Ralph SA, Ren Q, Sargeant TJ, Salzberg SL, Stoeckert CJ, Sullivan SA, Yamamoto MM, Hoffman SL, Wortman JR, Gardner MJ, Galinski MR, Barnwell JW, Fraser-Liggett CM: Comparative genomics of the neglected human malaria parasite Plasmodium vivax. Nature 2008, 455:757-763.

28. Aurrecoechea C, Brestelli J, Brunk BP, Dommer J, Fischer S, Gajria B, Gao X, Gingle A, Grant G, Harb OS, Heiges M, Innamorato F, lodice J, Kissinger JC, Kraemer E, Li W, Miller JA, Nayak V, Pennington C, Pinney DF, Roos DS, Ross C, Stoeckert CJ, Treatman C, Wang H: PlasmoDB: a functional genomic database for malaria parasites. Nucl Acids Res 2009, 37:D539-543.

29. Pain A, Bohme U, Berry AE, Mungall $K$, Finn RD, Jackson AP, Mourier $T$, Mistry J, Pasini EM, Aslett MA, Balasubrammaniam S, Borgwardt K, Brooks K, Carret C, Carver TJ, Cherevach I, Chillingworth T, Clark TG, Galinski MR, Hall N, Harper D, Harris D, Hauser H, Ivens A, Janssen CS, Keane T, Larke N, Lapp S, Marti M, Moule S, Meyer IM, Ormond D, Peters N, Sanders M, Sanders S, Sargeant TJ, Simmonds M, Smith F, Squares R, Thurston S, 
Tivey AR, Walker D, White B, Zuiderwijk E, Churcher C, Quail MA, Cowman AF, Turner CMR, Rajandream MA, Kocken CHM, Thomas AW, Newbold Cl, Barrell BG, Berriman M: The genome of the simian and human malaria parasite Plasmodium knowlesi. Nature 2008, 455:799-803.

30. Ip_solve: Open source (Mixed-Integer) Linear Programming system. [http:///psolve.sourceforge.net/].

31. Noble WS: How does multiple testing correction work? Nat Biotech 2009, 27:1135-1137.

32. Li L, Stoeckert CJ, Roos DS: OrthoMCL: Identification of Ortholog Groups for Eukaryotic Genomes. Genome Res 2003, 13:2178-2189.

33. Notredame C, Higgins DG, Heringa J: T-coffee: a novel method for fast and accurate multiple sequence alignment. J Mol Biol 2000, 302:205-217

34. Do CB, Mahabhashyam MSP, Brudno M, Batzoglou S: ProbCons:

Probabilistic consistency-based multiple sequence alignment. Genome Res 2005, 15:330-340.

35. Edgar RC: MUSCLE: multiple sequence alignment with high accuracy and high throughput. Nucl Acids Res 2004, 32:1792-1797.

36. LaCount DJ, Vignali M, Chettier R, Phansalkar A, Bell R, Hesselberth JR, Schoenfeld LW, Ota I, Sahasrabudhe S, Kurschner C, Fields S, Hughes RE: A protein interaction network of the malaria parasite Plasmodium falciparum. Nature 2005, 438:103-107.

37. Tuteja R: Genome wide identification of Plasmodium falciparum helicases: a comparison with human host. Cell Cycle 2010, 9:104-120

38. Beaussart F, Weiner J, Bornberg-Bauer E: Automated Improvement of Domain ANnotations using context analysis of domain arrangements (AIDAN). Bioinformatics 2007, 23:1834-1836.

39. Ekman D, Björklund ÅK, Elofsson A: Quantification of the Elevated Rate of Domain Rearrangements in Metazoa. J Mol Biol 2007, 372:1337-1348.

40. Wang Y, Sadreyev Rl, Grishin NV: PROCAIN: protein profile comparison with assisting information. Nucl Acids Res 2009, 37:3522-3530.

41. Karplus K, Karchin R, Barrett C, Tu S, Cline M, Diekhans M, Grate L, Casper J, Hughey $R$ : What is the value added by human intervention in protein structure prediction? Proteins 2001, 45:86-91.

42. Söding J: Protein homology detection by HMM-HMM comparison. Bioinformatics 2005, 21:951-960.

43. Bidargaddi NP, Chetty M, Kamruzzaman J: Hidden Markov Models Incorporating Fuzzy Measures and Integrals for Protein Sequence Identification and Alignment. Genomics, Proteomics \& Bioinformatics 2008 , 6:98-110.

44. Karlin S, Altschul SF: Methods for assessing the statistical significance of molecular sequence features by using general scoring schemes. P Natl Acad Sci USA 1990, 87:2264-2268.

45. Barrett C, Hughey R, Karplus K: Scoring hidden Markov models. Comput Appl Biosci 1997, 13:191-199.

doi:10.1186/1471-2105-12-90

Cite this article as: Ochoa et al:: Using context to improve protein domain identification. BMC Bioinformatics 2011 12:90.

\section{Submit your next manuscript to BioMed Central and take full advantage of:}

- Convenient online submission

- Thorough peer review

- No space constraints or color figure charges

- Immediate publication on acceptance

- Inclusion in PubMed, CAS, Scopus and Google Scholar

- Research which is freely available for redistribution

Submit your manuscript at www.biomedcentral.com/submit
C Biomed Central 